Noname manuscript No.

(will be inserted by the editor)

\title{
Optimized Herschel/PACS photometer observing and data reduction strategies for moving solar system targets*
}

Kiss, Cs. • Müller, Th.G. • Vilenius, E. •

Pál, A. · Santos-Sanz, P. · Lellouch, E. ·

Marton, G. · Verebélyi, E. · Szalai, N. •

Hartogh, P. · Stansberry, J. · Henry,

F. - Delsanti, A.

Received: date / Accepted: date

Abstract The "TNOs are Cool!: A survey of the trans-Neptunian region" is a
Herschel Open Time Key Program that aims to characterize planetary bodies
at the outskirts of the Solar System using PACS and SPIRE data, mostly taken
as scan-maps. In this paper we summarize our PACS data reduction scheme
that uses a modified version of the standard pipeline for basic data reduction,
optimized for faint, moving targets. Due to the low flux density of our targets
the observations are confusion noise limited or at least often affected by bright
nearby background sources at 100 and $160 \mu \mathrm{m}$. To overcome these problems
we developed techniques to characterize and eliminate the background at the
positions of our targets and a background matching technique to compensate
for pointing errors. We derive a variety of maps as science data products
that are used depending on the source flux and background levels and the

* Herschel is an ESA space observatory with science instruments provided by European-led Principal Investigator consortia and with important participation from NASA.

Cs. Kiss*, A. Pál, G. Marton, E. Verebélyi, N. Szalai

Konkoly Observatory, MTA CSFK

Tel.: +36-1-3919341

Fax: +36-1-2754668

E-mail: * pkisscs@konkoly.hu

Th. G. Müller and E. Vilenius

Max-Planck-Institut für extraterrestrische Physik, Garching, Germany

P. Santos-Sanz

Instituto de Astrofísica de Andalucía-CSIC, Granada, Spain

E. Lellouch, F. Henry and A. Delsanti

LESIA, Observatoire de Paris, Meudon, France

P. Hartogh

Max-Planck-Institut für Sonnensystemforschung, Katlenburg-Lindau, Germany

J. Stansberry

Space Telescope Science Institute, Baltimore, MD, USA 
scientific purpose. Our techniques are also applicable to a wealth of other Herschel solar system photometric observations, e.g. comets and near-Earth asteroids. The principles of our observing strategies and reduction techniques for moving targets will also be applicable for similar surveys of future infrared space projects.

Keywords Instrumentation:detectors:Herschel/PACS · Methods:observational · Techniques:photometric

\section{Introduction}

TNOs are frozen leftovers from the formation period of the outer Solar System. Due to their relatively small sizes and large distances little information can be earned from visual range observations only but physical characteristics can be derived using visual range and thermal emission observations together. The cold surface of planetary bodies at the outer regions of the Solar System (20$50 \mathrm{~K})$ made the photometric instruments of the Herschel Space Observatory (Pilbratt et al., 2010) very well suited to survey these populations at farinfrared and submillimetre wavelenghts. The "TNOs are Cool!": A survey of the trans-Neptunian region Herschel Open Time Key Program (Müller et al., 2009) used the PACS (Poglitsch et al., 2010) and SPIRE (Griffin et al., 2010) instruments to observe the thermal emission of trans-Neptunian objects and Centaurs. Due to the prominent sky background at these wavelengths, the observations are severely affected by confusion noise at the longer PACS photometer bands (100 and $160 \mu \mathrm{m})$ and the SPIRE bands $(250,350$ and $500 \mu \mathrm{m})$, where the resolving power is poorer. However, the relatively fast apparent motion of our targets (typically a few arcsecs per hour) made it possible to develop observation schemes and data reduction techniques that could fully exploit the potential of multi-epoch observations and eliminate the background (and hence the confusion noise) very effectively. Due to the high sensitivity of PACS in this programme - in agreement with our original goals - we were able to sample about $10 \%$ of the known TNOs and determine their main physical characteristics (size, albedo, surface properties) with the synergy of ground-based optical and space-born far-infrared data. The main science goals of our program were (i) simultaneous measurement of size and albedo of a large sample of targets; (ii) Determination of densities of binary TNOs based on measured effective sizes; (iii) Constraining of thermal and surface properties; (iv) Measurement of thermal lightcurves of few objects by continuously observing them throughout an entire rotational period. Our science results are published in a series of about 15 publications, including recent ones by Santos-Sanz et al. (2012), Mommert et al. (2012), Vilenius et al. (2012), Pal et al. (2012), Kiss et al. (2013), Fornasier et al. (2013), Lellouch et al. (2013), and two recently submitted papers by Duffard et al. (2013), and Vilenius et al. (2013). The large amount and high complexity of our data required the development of our own pipeline processing, and some special data reduction techniques were developed to fully exploit the capabilities of 
our multi-epoch observations. In this paper, in addition to the description of our original observation planning strategies, we summarize the main steps of our PACS scan-map data reduction and the validation of the processing scheme used in our programme. We also describe our data products that are derived from the raw science data and that are used to obtain the final flux densities.

\section{Observations}

\subsection{Open Time Key Program and related observations}

The observations of the Herschel key programme "TNOs are Cool: A survey of the trans-Neptunian region" Herschel Open Time Key Program (Müller et al., 2009) consist of Science Demonstration Phase (SDP) and a Routine Science Phase observations, with a total time allocation of 403.3 hours (including all overheads), of which 30.6 hours were used in the SDP between November 2009 and January 2010, and all observations were performed before by October 2012. All SDP targets were observed again during the routine phase. We used $95 \%$ of the total time for photometry observations with PACS $(60-210 \mu \mathrm{m})$ and the rest with SPIRE $(200-670 \mu \mathrm{m})$. The data reduction of our SPIRE observations is presented in Fornasier et al. (2013).

In addition to the OTKP measurements, there were several successful Open Time Programs and Director's Discretionary Time (DDT) observations related to the "TNOs are Cool!" OTKP. For example, Eris and Quaoar thermal light curves were obtained in the open time program "Probing the extremes of the outer Solar System: short-term variability of the largest, the densest and the most distant TNOs from PACS photometry" (PI: E. Vilenius; see Kiss et al., 2012), we investigated "Pluto's seasonal evolution and surface thermal properties" (PI: E. Lellouch), and observed two objects that move on peculiar orbits, $2012 \mathrm{DR}_{30}$ and $2013 \mathrm{AZ}_{60}$ in two dedicated DDTs in May 2012 and April 2013 , respectively (see Kiss et al., 2013 for the $2012 \mathrm{DR}_{30}$ results). All these observations used the same reduction pipeline to obtain the final flux densities of the targets. More details on these additional observations are presented in the respective papers. A summary of all "TNOs are Cool!" Open Time Key Program observations can be found at the following webpage:

\section{http://kisag.konkoly.hu/tnodatareductionsummary}

\subsection{Observation design}

We used the Standard Thermal Model (Lebofsky et al., 1986, and references therein) to predict flux densities of our targets in the PACS bands. Based on earlier Spitzer work (Stansberry et al., 2008) we adopted a geometric albedo of 0.08 for those targets which did not have previous Spitzer results, and used a hybrid-STM, in which the beaming parameter differs from the canonical STM 
beaming parameter. We used $\eta=1.25$ (Stansberry et al., 2008) for observation planning purposes. The predicted thermal fluxes depend on the sizes, which are connected to the assumed geometric albedo and the absolute V-magnitudes via

$$
D=\frac{2 a}{\sqrt{p_{V}}} \times 10^{\frac{1}{5}\left(m_{\odot}-H_{V}\right)},
$$

where $D$ is the area equivalent diameter of the TNO assumed to be spherical, $a$ is the distance of one astronomical unit, $p_{V}$ is the assumed geometric albedo, $m_{\odot}$ is the apparent $\mathrm{V}$-magnitude of the Sun, and $H_{V}$ is the absolute $\mathrm{V}$-magnitude of the TNO.

The submitted and accepted OTKP target list consisted of 137 TNOs and Centaurs, and in addition the giant planet moons Phoebe and Sycorax. We have observed 132 of them, including the two moons. The main reasons for not observing seven targets were too uncertain astrometry and too low predicted flux. The absolute $\mathrm{V}$-magnitudes $\left(H_{\mathrm{V}}\right)$ used in the planning of our sample were $<10.8 \mathrm{mag}$ ( $<8.2 \mathrm{mag}$ if Centaurs are excluded). In the scientific analysis of observed targets we use the latest optical photometry available in the literature or in data bases, or determine absolute visual magnitudes based on data from these sources. For some targets, there has been a significant change in $H_{\mathrm{V}}$ compared to the estimate used during the planning.

The observation duration and timing constraints have been planned individually for each target. All of the targets observed had predicted astrometric $3 \sigma$ uncertainties less than $10^{\prime \prime}$ at the time of the Herschel observations (David Trilling, priv. comm.). In the routine phase we used the AstDys web servic $\mathbb{1}^{1}$

\subsection{Observing modes}

We specified the astronomical observation requests (AOR) in HSpot, a tool provided by the Herschel Science Ground Segment Consortium. In the PACS photometer AORs a selection is made to observe either the blue+red or the green+red channels (the red channel data are taken simultaneously whichever short wavelength filter is chosen). The sensitivity of the blue channel is usually limited by instrument noise, while the red channel is confusion-noise limited (Poglitsch et al., 2010). The sensitivity in the green channel can be dominated by either of them, depending on the depth and the region of the sky of the observation. We optimized the timing of the observations by selecting the visibility window in which the far-infrared confusion noise (Kiss et al., 2005) was the lowest in the green channel.

Since all of our targets, including complete multiple TNO systems, have apparent sizes smaller than the PACS spatial resolution, we aimed to measure the disk-integrated flux density using either the point-source mode (usually referred to as "chop-nod" mode) or the mini-scan map mode. Both options

1 Asteroids Dynamic Site by A. Milani, Z. Knezevic, O. Arratia et al., http://hamilton.dm.unipi.it/astdys/ calculations based on the OrbFit software 
were tested extensively during the science demonstration phase (SDP) of the Herschel mission.

Chop-nod mode: Details on the chop-nod mode can be found in the PACS Observer's Manua ${ }^{2}$ and in the observing mode release not ${ }^{3}$ In this mode the target is moved to different locations on the detector array by a chopper mirror as well as by nodding the telescope pointing. The source remains on the array all the time. One of the few parameters left for the astronomer to choose, in addition to the number of repetitions, is whether dithering is used. We used the PS mode $(10,16$ or 36 repetitions corresponding to durations of $0.4,0.7$ or 1.6 hours) with dithering only for six targets during the SDP (Müller et al., 2010 $0^{4}$ Lim et al., 201 ${ }^{5}$. The flux densities of our targets observed in this mode were derived with the standard chop-nod reduction pipeline script available in HIPE (Ott 2010), using the latest available version. The final chop-nod mode maps contain images of the target, as well as images of all background sources in the neighbouring field, forming a specific structure. This makes it very challenging to identify the target and perform suitable photometry in most cases, especially for faint sources close to the confusion limit. As the usability of this mode was very much restricted according to our tests in the SDP, and as the mini scan-maps showed a much better overall efficiency (as discussed in detail below), at the end of the SDP we decided to switch to the scan-map mode as our default observing mode. Note that all of our chop-nod SDP targets were later re-observed in the routine phase in the mini-scan map mode. The chop-nod measurements were reduced using the latest version of the standard pipeline, and no further combined products were derived from the Level-2 chop-nod maps. Since the chop-nod mode is well calibrated, most recently by Nielbock et al. (this issue), chop-nod data can be used in radiometric modeling techniques in combination with the mini-scan map data taken at a different epoch. Science results using chop-nod data of the "TNOs are Cool!" Open Time Key Program have been published in Müller et al. (2010) and Lim et al. (2010).

mini scan-maps: During the SDP we tested the mini scan-map mode ${ }^{6}$ with two small maps per target, observed at different scan angles with respect to the detector array. The SDP light curves observations of (136108) Haumea were also performed in this mode (Lellouch et al. 2010). This mini-scan flavor of the scan-map mode also became the recommended mode for faint point sources (Poglitsch et al., 2010) due to its better overall efficiency in the case of relatively faint targets, such as ours. In this mode the pointing of the telescope

\footnotetext{
2 http://herschel.esac.esa.int/Docs/PACS/html/pacs_om.html

3 http://herschel.esac.esa.int/twiki/pub/Public/PacsCalibrationWeb/PhotMiniScan_ReleaseNote_20101112.pdf

4 Targets: 42355 Typhon (two observations), 79360 Sila, 82075 (2000 YW 134 ), 126154 $\left(2001 \mathrm{YH}_{140}\right)$ and $208996\left(2003 \mathrm{AZ}_{84}\right)$.

5 Target 136472 Makemake (two observations).

6 http://herschel.esac.esa.int/twiki/pub/Public/PacsCalibrationWeb/ PhotMiniScan_ReleaseNote_20101112.pdf
} 

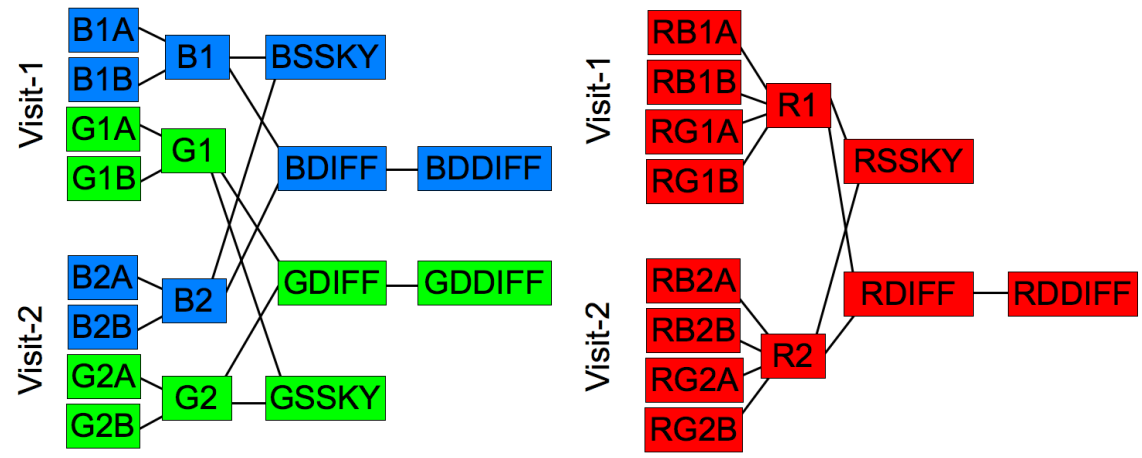

Fig. 1 Outline of our standard observing and image derivation scheme. The single maps (first column) are combined to obtain the co-added, single visit maps (second column), and these co-added maps are used to produce the different science data products (SSKY, DIFF and DDIFF maps) that are used to obtain the final fluxes. The left and right panels of the figure show the scheme separately for the short wavelength $(70 / 100 \mu \mathrm{m}$, or blue/green) and for the long wavelength $(160 \mu \mathrm{m}$ or red) PACS channels. In each box in the first letter marks the filter $(\mathrm{B}=$ blue, $\mathrm{G}=$ green, $\mathrm{R}=\mathrm{red})$, the second marks the epoch $(1=$ Visit- $1,2=\mathrm{Visit}-2)$, the third marks the scan direction $(A=70 \mathrm{deg}, \mathrm{B}=110 \mathrm{deg})$. In the case of the red filter sequences the double letters (RB or RG) marks the corresponding short/long wavelength filter combination. The SSKY, DIFF and DDIFF labels correspond to the supersky-subtracted, differential and double-differential images, respectively.

is slewed at a constant speed over parallel lines, or "legs". We used 10 scan legs in each AOR, separated by $4^{\prime \prime}$. The length of each leg was $3.0^{\prime}$ except during the SDP and in the beginning of the routine phase, when $3.5^{\prime}$ and $2.5^{\prime}$ were used. The selected slewing speed was $20^{\prime \prime} \mathrm{s}^{-1}$ except for 22 AORs in January 2010 when the fast scan speed of $60^{\prime \prime} s^{-1}$ was tested. During the SDP different observing strategies were in use but in the routine phase we mostly used a constant sequence of AORs (except for lightcurve observations). For a given channel selection (blue or green) we grouped pairs of AORs, with scan orientations of $70^{\circ}$ and $110^{\circ}$ with respect to the detector array (Scan-A and Scan-B), in order to make optimal use of the rectangular shape of the detector. Thus, during a single visit of a target we grouped 4 AORs to be observed in sequence: two AORs in different scan directions with the same short wavelength PACS filter, and then this sequence repeated for the second short wavelength channel. The outline of this scheme is presented in Fig. 1 .

Within each AOR the observation of the mini-map was repeated from one to six times, depending on the predicted flux of the target, as described above. Each target was visited twice with similar AORs repeated in both visits. The timing of the visits (Visit-1 and Visit-2) was such that the target had moved $30-50^{\prime \prime}$ between the visits so that the target position during the second visit is within the high-coverage area of the map from the first visit. This allows us to determine the background for the two source positions, as the Visit-1 and Visit-2 images serve as mutual backgrounds. The typical time difference between Visit-1 and Visit-2 was in the order of a few hours for Centaurs and 1-1.5 days for TNOs due to their different apparent velocities. In the case of 
fast moving near-Earth objects we could use individual repetitions within the same measurement as mutual backgrounds in two or more visits, as was done e.g. in the case of (162173) $1999 \mathrm{JU}_{3}$ (O'Rourke et al., 2012).

\section{Data reduction of mini scan-maps}

We use a modified version of the PACS pipeline for basic data reduction of scan-map ${ }^{7}$ (producing single images per OBSID) from raw data to Level2 maps (for the definition of the Herschel/PACS data product levels, see the PACS Observer's Manual). We applied the following main parameters in HIPE (for a summary of the PACS photometer scan-maps calibration, see Balog et al., this issue):

- Slews are selected on scan speed, usually between 15 and $25^{\prime \prime} s^{-1}$ (our maps are predominantly observed with $20^{\prime \prime} s^{-1}$ scan speed).

- High-pass filter width of 8, 9 and 16 are used at 70, 100 and $160 \mu \mathrm{m}$, respectively (high pass filter width sets the number of frames $[2 n+1]$ used for median subtraction from the detector timeline; see Popesso et al., 2012 and Balog et al., 2013, this issue, for a detailed description of the method)

- Masking pixels above 2-sigma, and at the source position with 2xFWHM radius

- We apply second level deglitching with nsigma=30, the sigma-clipping parameter of this deglitching method working on the map level (see the PACS Data Reduction Guide for more details). At the same time, the multiresolution median transform (MMT) deglitching task is disabled (see Vavrek et al., 2008).

- Despite that we observe solar system targets, we do not correct for the apparent motion of target to obtain the data products described below. In most cases the apparent displacement of our target during a single visit is notably smaller than the blue (shortest wavelength) FWHM, and such a correction would degrade the performance of our background elimination techniques at the highest spatial frequencies. For those targets that have a displacement larger than $0.5^{\prime \prime}$ per individual visit, we use the standard, proper motion corrected reduction scheme.

We apply the drizzle method to project the time-line data and produce the single maps using the photProject() task in HIPE, with a pixel fraction parameter of 1.0 in most cases. We use default pixel sizes of $1^{\prime \prime} .1,1^{\prime \prime} 4$ and $2^{\prime \prime} 1$ in the PACS 70, 100 and $160 \mu \mathrm{m}$ bands, respectively (in some special cases different pixel sizes or pixel fraction parameters were also used).

The data reduction is performed on dedicated computers which have the sufficient amount of memory (up to $128 \mathrm{~GB}$ ) and the necessary CPU performance. These computers are located at Max-Planck-Insititut für Sonnensystemforshung (Katlenburg-Lindau, Germany) and Konkoly Observatory (Budapest, Hungary).

\begin{tabular}{ccccc}
\hline 7 For details, see the PACS Data Reduction & Guide: \\
http://herschel.esac.esa.int/twiki/bin/viewfile/Public/PacsCalibrationWeb?rev=1 filename=PDRG_Dec2011.pdf
\end{tabular}




\section{4 "TNOs are Cool!" image products}

As a further step in our data reduction, we combine the single maps obtained in Visit-1 and Visit-2 with the aim to reduce the effect of the background as much as possible. We produce the following image products:

- Co-added images (from the Scan-A and Scan-B images of the same, single visit)

- Differential images (from the co-added images, DIFF). Optimal coordinate offsets are determined with the "background matching" method

- Super-sky subtracted images (from the co-added images, SSKY)

- Double differential (DDIFF) images, created from the differential images, using "source matching" to determine the ideal offsets

We are summarizing the details of these products and the necessary intermediate steps below.

Co-added images: Co-added images are generated using the maps of the individual OBSIDs in a specific band and in a single visit. In the case of both the blue and the green band we co-add two maps, the Scan-A and Scan-B images $(\mathrm{B} 1=\mathrm{B} 1 \mathrm{~A}+\mathrm{B} 1 \mathrm{~B}, \mathrm{G} 1=\mathrm{G} 1 \mathrm{~A}+\mathrm{G} 1 \mathrm{~B}$, etc., according to the scheme presented in Fig. 1). In the red band, all the four red maps (taken in parallel with blue/green and scan/cross-scan) are co-added ( $\mathrm{R} 1=\mathrm{RB} 1 \mathrm{~A}+\mathrm{RB} 1 \mathrm{~B}+\mathrm{RG} 1 \mathrm{~A}+\mathrm{RG} 1 \mathrm{~B}$, etc.). This processing step is performed in IDL $^{8}$ considering the coverage values of each pixel as weights. This, in principle, is identical with the co-added images obtained using the MosaicTask() function in HIPE. These co-added images are the bases of the further processing steps and data products.

Supersky-subtracted images: To create the so-called supersky-subtracted images first a background map is generated using the single maps. To do this we mask the target in each single map and co-add the maps in the sky coordinate system. This step produces a background map without the target. The background map is subtracted from the single maps producing backgroundsubtracted single maps. Finally the background-subtracted maps are co-added in the target frame, producing the final combined map on which photometry is performed (this method has previously been described in detail and demonstrated with sample images in Santos-Sanz et al., 2012). A feature of this method is that at the masked locations the signal-to-noise ratio is lower than at the other parts of the image, since only the data of a single visit can be used here. An example is presented in Fig. 2 for the Centaur $2002 \mathrm{GZ}_{32}$. This background subtraction technique was originally developed for the Spitzer/MIPS observations of trans-Neptunian objects (Stansberry et al. 2008), and we kept the original scheme to obtain the final images, but applied the background matching method to correct for coordinate frames offsets between the two visits (see below).

\footnotetext{
8 Interactive Data Language, Research Systems Inc.
} 


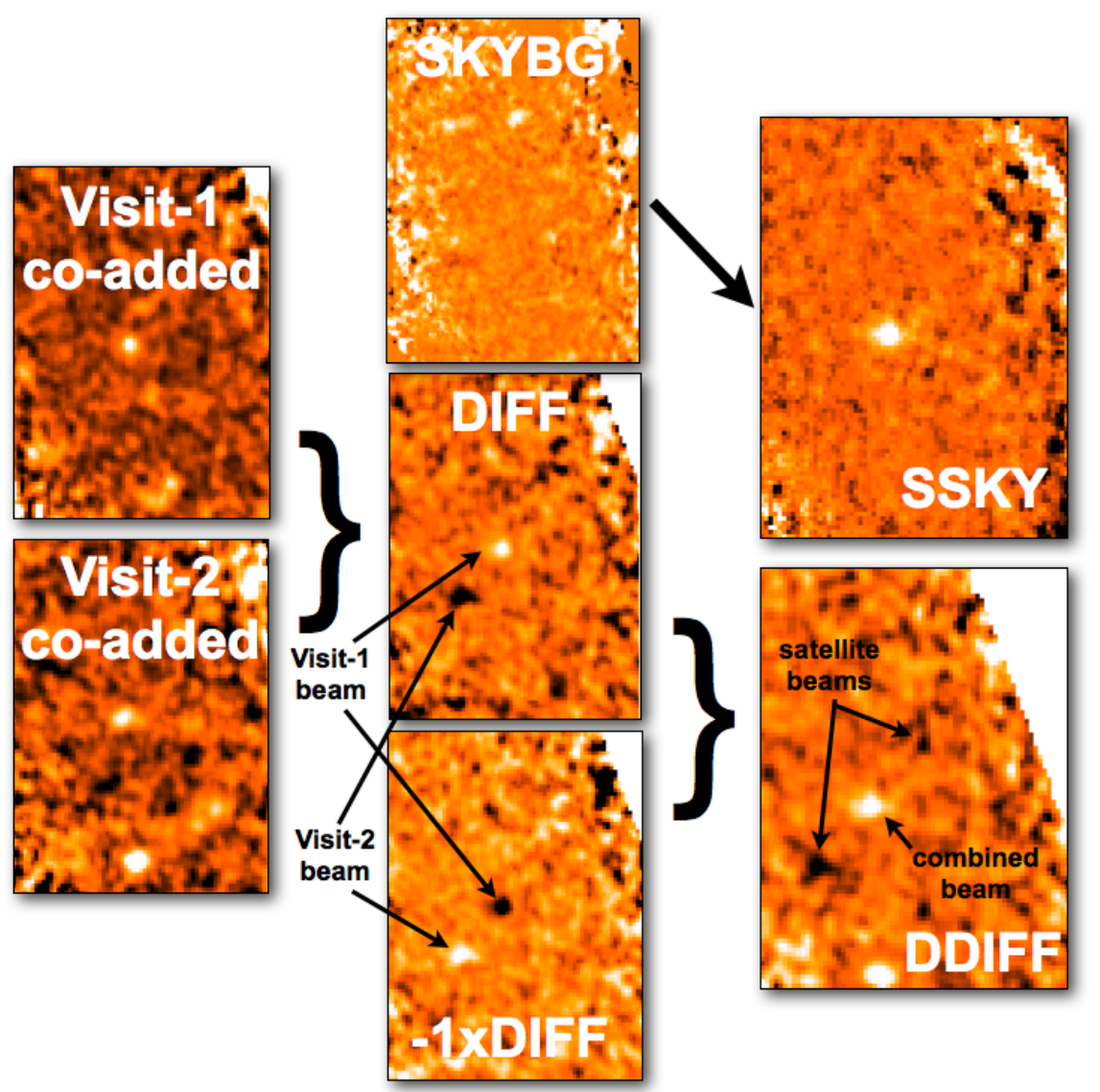

Fig. 2 Demonstration of mini-scan map image processing steps and products from the coadded image level with the $160 \mu \mathrm{m}$ images of the Centaur $2002 \mathrm{GZ}_{32}$, a relatively faint target in this band (see also Fig. 1 and Sect. 4 for the detailed data reduction scheme)

Differential images and background matching: Background matching is used to correct for the small offsets in the coordinate frames of the Visit-1 and Visit-2 images when obtaining the differential image, which is simply the difference of the combined Visit-1 and Visit-2 images in the respective bands $(\mathrm{BDIFF}=\mathrm{B} 1-\mathrm{B} 2$, etc., see also Fig. 1). Incorrect offsets can easily be identified by the appearance of positive/negative spot pairs (see the left panel of Fig. 3 above, marked with black ovals) - these are completely eliminated on the corrected image (right panel of the same image). The offset to be applied can be determined using images of systematically shifted coordinate frames and then determining the offset which provides the smallest standard deviation of flux values in a pre-defined coverage interval (typically $0.3<$ coverage $<0.9$, see the contour map of Fig. 3). Our tests have proved that the same offset is obtained using any of the three PACS bands, however, in most cases the offset 

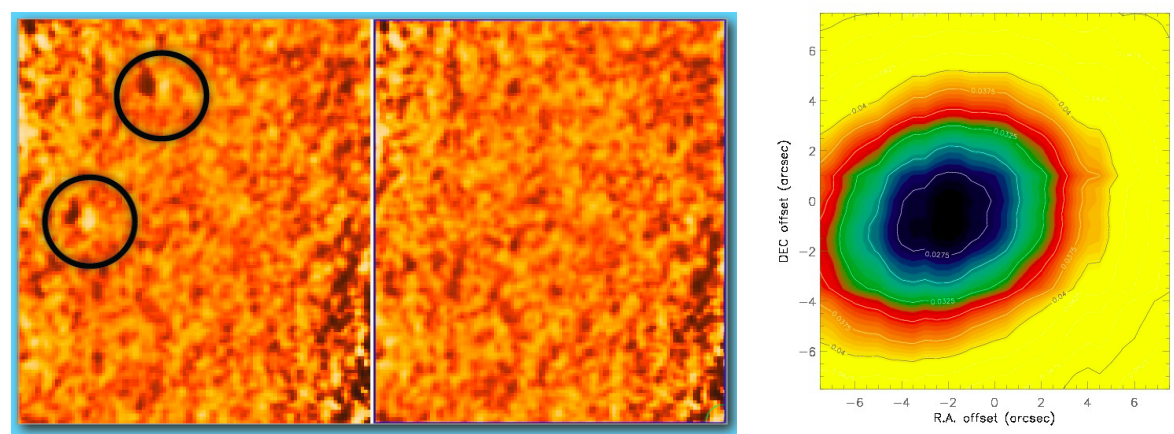

Fig. 3 Left panel: Original (left) and background matched (right) differential images of the same pair of $70 \mu \mathrm{m}$ images. Right panel: Contour map of residual noise as a function of coordinate offsets. The optimal offsets were $-2 . \prime 5$ and $-1{ }^{\prime \prime} 0$ in R.A. and DEC, respectively.

can be most readily determined using the $160 \mu \mathrm{m}$ images, due to the strong sky background w.r.t. the instrument noise.

Double-differential images: A double-differential (DDIFF) image is made of the DIFF image of a target at a specific wavelength. The disadvantage of the DIFF image is that the images of the target appears as two separated beams (one positive and one negative), corresponding to the two visits. To produce a DDIFF image, first the DIFF image is "folded" (multiplied by -1 ). The folded image is shifted in a way that the location of the two positive beams of the target match on the original and the folded image (see Fig. 22). Then, the original and the folded/shifted DIFF images are co-added:

$$
\operatorname{DDIFF}(\underline{x})=\operatorname{DIFF}(\underline{x})-\operatorname{DIFF}(\underline{x}+\underline{\theta})
$$

where the optimal offset $\underline{\theta}$ is determined with the source matching method (see below). The DDIFF image contains a positive beam with the total flux of the target and two negative beams at the sides with "half" of the total flux 9 It is a clear advantage of this method that the photometry can be performed on a single beam, and one does not have to combine the flux of two beams as in the case of the DIFF images. In the case of the DDIFF images the noise is increased by a factor of $\sqrt{2}$ when compared to the corresponding DIFF image, and flux variations between the two visits are flattened out. However, the signal-to-noise of the target is improved by $\sqrt{2}$ with respect to a single DIFF image which is very important in detecting faint targets. This method has proved to provide the best performance in the detection of very faint sources $(<2 \mathrm{mJy}$ at $70 \mu \mathrm{m})$, superior to the DIFF or SSKY images.

\footnotetext{
9 well, this is not exactly half. The two negative beams contain the half flux of the first and second visits separately, which is half the co-added DDIFF flux only if the flux of the target has not changed between the two visits
} 

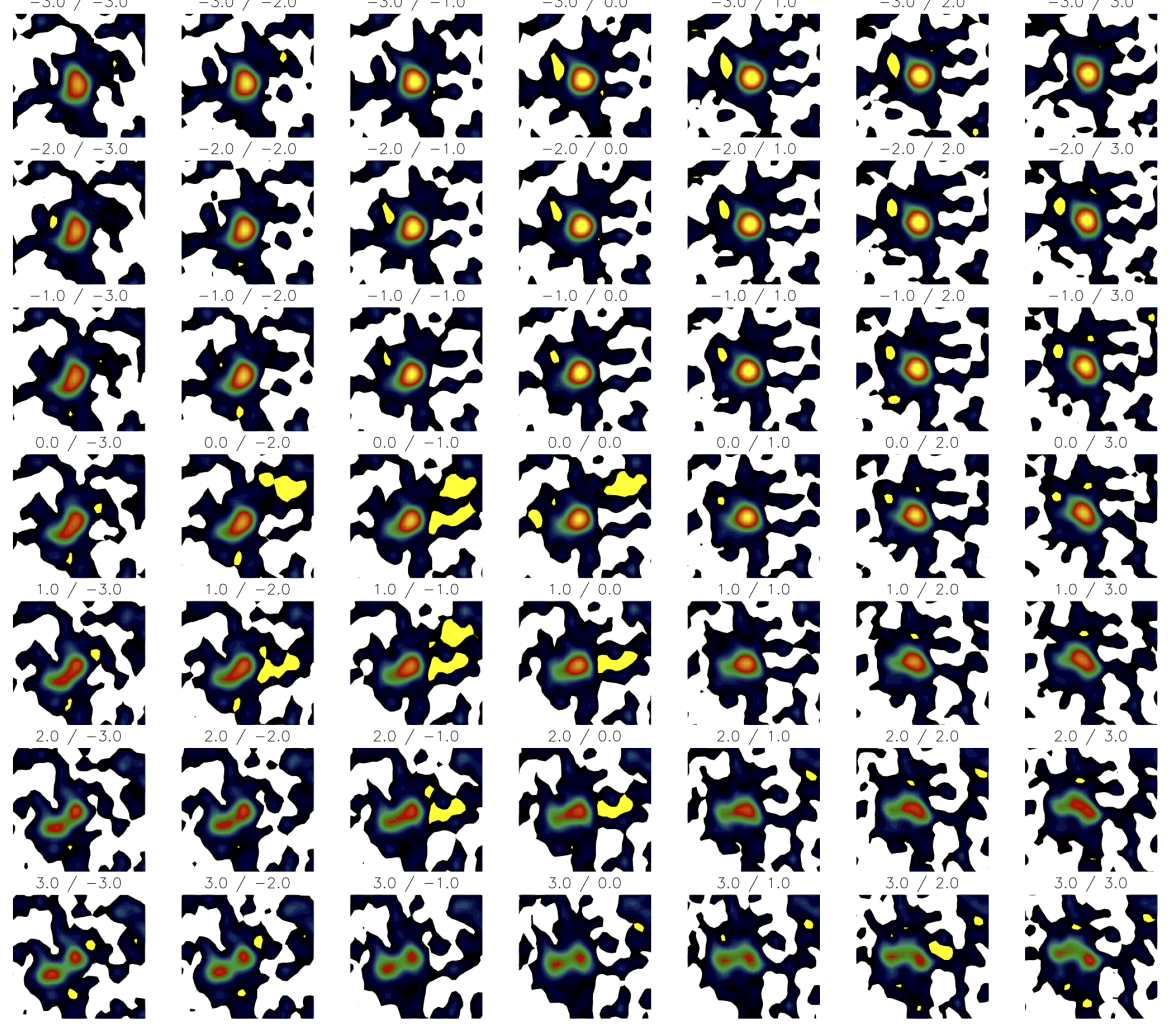

. 4 Source matching for Ixion (green band) to determine the optimal offset for the final DDIFF image. Wrong offsets can easily be identified by the distorted or double-peaked shape of the combined image. The numbers above the stamp figures correspond to the actual offsets in arcsec units (the optimal offsets are $-22^{\prime \prime} 0$ and $+1^{\prime \prime} .0$ in this case).

Source matching: Background matching (see above) provided offsets for coordinate frame differences in the two visits, but positional differences may still remain due to e.g. not well known positions of the target, and wrong offsets lead to distorted shapes of the target image when the images of the two visits are combined to obtain double-differential images. "Source matching" determines the optimal offset $(\underline{\theta})$ that the original and folded DIFF images have to be shifted with to obtain the best matching of the centroids of the targets when we combine them to produce the DDIFF images. Typical offsets are a few arcseconds, we use the $\pm 4^{\prime \prime}$ range both in R.A. and DEC to determine the offset. We demonstrate the method in Fig. 4 for Ixion. For relatively bright targets (a few tens of mJy) the source matching correction typically increase the flux by $\lesssim 10$ per cent compared to the uncorrected case - in these cases the optimal offsets are in the order of $2^{\prime \prime}$. However, to detect very faint targets, source matching is a necessary step to detect the target at all. 
The source matching optimized double-differential method has proved to provide the best performance in the detection of very faint sources $(\lesssim 2 \mathrm{mJy}$ at 70 and $100 \mu \mathrm{m}$ and $\lesssim 5 \mathrm{mJy}$ at $160 \mu \mathrm{m}$ ), superior to the DIFF or SSKY images. The clear difference between the DIFF and DDIFF images is the $\sqrt{2}$ signal-to-noise improvement. Similarly, in the case of SSKY images the noise level is higher at the masked area in the vicinity of the target, as only the background information of a single visit is used. For moderately bright targets (at least a few mJy flux density) both the DDIFF and SSKY products are used to extract fluxes.

An automatic derivation of the combined Open Time Key Program data products is performed using the FITSH package (Pál, 2012).

\section{Photometry}

Aperture photometry of our targets can be performed using various tools (IDL/DAOPHOT, IRAF, HIPE) that provide practically identical results, as it has been tested at the early phases of our programme. As the default image pixel size is adjusted to the actual FWHM in all PACS bands (1.'1, 1."4 and 2 '. 1 in the blue, green and red bands, respectively), an ideal aperture of 4-5 pixels in radius has been identified in all bands using flux growth curves of several targets. The encircled energy fraction coefficients - that correct for the flux outside the measuring aperture - are taken from the PACS Observer's Manual.

Absolute photometric accuracy Although the final, level-2.0 images after the pipeline processing are already flux calibrated, it is important to check whether our processing introduces any discrepancies with respect to the official flux calibration. It is especially important in our case since the flux calibration of the PACS photometer is based on a few bright stars and asteroids, and no faint star (in the order of $\sim 100 \mathrm{mJy}$ at $70 \mu \mathrm{m}$ or below) is used officially as a calibrator. Our targets are significantly fainter than the calibrator objects, and in principle they may require a different flux calibration. We selected a few faint star calibration measurements with available photospheric flux predictions for the PACS 70, 100 and $160 \mu \mathrm{m}$ bands (HD 139669: Shirahat et al., 2009; $\gamma$ Dra, HD 170693 and HD 138265: Gordon et al., 2009). The measurement were taken from the Herschel Archive and processed in the same way as would be done with the TNO measurements. After the level-2.0 products (maps) are created, we performed aperture photometry again on the central source in the same way as in the case of the TNO measurements. We characterise the absolute calibration accuracy by two numbers: a coefficient that the measured flux has to be multiplied with to obtain the predicted flux $\left(\mathrm{F}_{\text {pred }}=\mathrm{r}_{\text {cal }} \times \mathrm{F}_{\text {meas }}\right)$ and the relative uncertainty of the measured to predicted fluxes $\left(\sigma_{c a l}\right)$. The $\mathrm{r}_{c a l}$ values we obtained are 1.03, 1.01 and 0.98 for blue, green and red bands, respectively, while the $\sigma_{c a l}$ values are $0.9 \%, 1.5 \%$ and $5.6 \%$, in very good agreement 
with the generaly quoted 5\% absolute accuracy of the PACS photometer flux calibration.

The absolute photometric accuracy of our full processing scheme - that includes differential, double differential or supersky-subtracted images - cannot be tested entirely using standard star measurements, as these objects do not move with respect to the background, making these types of images meaningless to produce. As the correctness of the flux calibration was approved in the first step (see above), in a second step we used the scan-map measurements of some bright TNOs and Centaurs (Quaoar, Orcus, Ixion and Chariklo, among others) to test whether flux densities are kept during the generation of our combined data products from the differential images to the double differential or supersky-subtracted images. The requirement was that, as the background is negligible for these bright targets, the average flux densities obtained at the original, single visit images must be the same as that of the combined products within the photometric uncertainties. According to these tests the combined data products fulfil this requirement (relative accuracies are better then $5 \%$ in all PACS bands).

Photometric uncertainty is determined using the "implanted source" method in the case of all data products. In this method we place 200 artificial sources on the image (a single one at a time) and this artificial source has a spatial flux distribution shape of the PACS PSF in the actual band. Then the same type of aperture photometry is performed on each implanted source as on the target. The sources are placed in regions with coverage values within a fixed interval (typically $0.3<$ coverage $<0.9$ ) - this excludes the vicinity of the target as well as the edges of the image where the coverage is low and therefore the noise is high. The photometric uncertainty of the map is taken as the standard deviation of the distribution of the artificial, implanted source fluxes (the distribution of these fluxes turned out to be very close to Gaussian in all cases).

\section{Detection statistics}

In the "TNOs are Cool!": A Survey of the trans-Neptunian region Herschel Open Time Key Program we observed 132 targets, in total 1131 observations (1089 in "KPOT_thmuelle_1", 2 in "AOTVAL_thmuelle_2" and 40 in "SDP_thmuelle_3"). A good majority of the targets $(>90 \%)$ are detected in at least one band (in the blue band in almost all cases), about $50 \%$ of them are detected in all the three PACS bands. With our techniques we managed to reach $0.6,0.9$ and $1.6 \mathrm{mJy}$ flux uncertainties using the combined products (DDIFF images) of 5-repetition single maps in the 70, 100 and $160 \mu \mathrm{m}$ PACS bands, respectively. 


\section{Summary}

In this paper we have demonstrated that the observation planning, data reduction pipeline and the related combined data products that we use in our open time key program are very effective tools to observe faint, moving solar system targets. Our methods, including observation planning, target selection, observing template setup, data reduction and product generation, could also be used to observe moving Solar System targets in future infrared space programs, like the SPICA mission (Nakagawa et al., 2012), to be launched in 2020. The applicability of these techniques for SPICA observations is summarized in Kiss et al. (2013b). More information on our open time key program can be found at the following webpage:

http://www.mpe.mpg.de/ tmueller/tno_public/index.htm

Acknowledgements This work has been supported by the Hungarian Research Fund (OTKA) grant K 104607, the PECS-98073 grant of the European Space Agency (ESA) and the Hungarian Space Office and the Bolyai Research Fellowship of the Hungarian Academy of Sciences. E.V. acknowledges the support of the German DLR project \# 50 OR 1108. The work of A.P. has been supported by the Lendület grant \# LP2012-31/2012 of the Hungarian Academy of Sciences. We are indebted to the Herschel observation planning team for their enthusiastic work during the active phase of the program which has highly contributed to the great success of the observations. We also thank the referee for providing constructive comments and help in improving this paper.

\section{References}

1. Balog, Z., Müller, T.G., Nielbock, M. et al., The Herschel-PACS photometer calibration: Point-source flux calibration, Exp. Astronomy, this issue (2013)

2. Fornasier, S., Lellouch, E., Müller, T., et al., "TNOs are Cool": A survey of the trans-Neptunian region. VIII. Combined Herschel PACS and SPIRE observations of 9 bright targets at 70-500 micron, A\&A, in press (2013)

3. Gordon, K.D., Engelbracht, C.W., Fadda, D., Absolute Calibration and Characterization of the Multiband Imaging Photometer for Spitzer. II. $70 \mu \mathrm{m}$ Imaging, PASP, 119, 1019 (2007)

4. Griffin, M. J., Abergel, A., Abreu, A., et al., The Herschel-SPIRE instrument and its in-flight performance, A\&A, 518, L3 (2010)

5. Kiss, Cs., Klaas, U., Lemke, D., Determination of confusion noise for far-infrared measurements, A\&A, 430, 343 (2005)

6. Kiss, Cs., Vilenius, E., Müller, Th.G., Thermal Emission of the Eris Dysnomia System as Observed by Herschel/PACS, Proceedings of the conference Asteroids, Comets, Meteors 2012, LPI Contribution No. 1667, id.6357 (2012)

7. Kiss, Cs., Szabó, Gy., Horner, J., et al., A portrait of the extreme Solar System object $2012 \mathrm{DR}_{30}, \mathrm{~A} \& \mathrm{~A}, 555, \mathrm{~A} 3$ (2013a)

8. Kiss, Cs., Müller, Th.G., Vilenius, E., et al., The trans-Neptunian objects after Herschel - What could SPICA add to the understanding of the Kuiper belt? Proceedings of the SPICA International Science Conference, PV-1. (2013b)

9. Lebofsky, L.A., Sykes, M.V., Tedesco, E.F., et al., A refined 'standard' thermal model for asteroids based on observations of 1 Ceres and 2 Pallas, Icarus, 68, 239 (1986)

10. Lellouch, E., Kiss, Cs., Santos-Sanz, P., et al., "TNOs are cool": A survey of the trans-Neptunian region. II. The thermal lightcurve of (136108) Haumea, A\&A, 518, L147 (2010) 
11. Lellouch, E., Santos-Sanz, P., Lacerda, P., "TNOs are Cool": A survey of the transNeptunian region. IX. Thermal properties of Kuiper belt objects and Centaurs from combined Herschel and Spitzer observations, A\&A, 557, A60 (2013)

12. Lim, T. L., Stansberry, J., Müller, T. G., et al., "TNOs are Cool": A survey of the trans-Neptunian region. III. Thermophysical properties of 90482 Orcus and 136472 Makemake, A\&A, 518, L148 (2010)

13. Mommert, M., Harris, A.W., Kiss, Cs., et al., "TNOs are Cool": A survey of the trans-Neptunian region. V. Physical characterization of 18 Plutinos using Herschel-PACS observations, A\&A, 541, A93 (2012)

14. Müller, T.G., Lellouch, E., Böhnhardt, H., et al., "TNOs are Cool: A Survey of the Transneptunian Region, EM\&P, 105, 209 (2009)

15. Müller, T.G., Lellouch, E., Stansberry, J., et al., "TNOs are Cool": A survey of the trans-Neptunian region. I. Results from the Herschel science demonstration phase (SDP), A\&A, 518, L146 (2010)

16. Müller, Th.G., Vilenius, E., Santos-Sanz, P. et al., TNOs are Cool: A survey of the trans-Neptunian region - Herschel observations and thermal modeling of large samples of Kuiper belt objects, European Planetary Science Congress 2012, id. EPSC2012-496 (2012a)

17. Müller, T.G., O'Rourke, L., Barucci, A. M., et al., Physical properties of OSIRISREx target asteroid (101955) 1999 RQ36. Derived from Herschel, VLT/ VISIR, and Spitzer observations, A\&A, 548, A36 (2012b)

18. Müller, T. G., Balog, Z., Nielbock, M. et al. 2013, Herschel celestial calibration sources: Four large main-belt asteroids as prime flux calibrators, Exp. Astronomy, this issue

19. Marton, G., Vavrek, R., Kiss, Cs., Müller, T.G., Photometric capabilities of the boloSource() algorithm, Exp. Astronomy, this issue (2013)

20. Moór, A., Müller, T.G., Kiss, C., et al., PACS photometer calibration block analysis, Exp. Astronomy, this issue (2013)

21. Nakagawa, T., Matsuhara, H., Kawakatsu, Y., The next-generation infrared space telescope SPICA, Proceedings of the SPIE, Vol. 8442, id. 84420 O (2012)

22. Nielbock, M., Müller, T.G., Balog, Z. et al., 2013, The Herschel-PACS photometer calibration: A time dependent flux calibration for the PACS chopped photometry AOT mode, Exp. Astronomy, this issue (2013)

23. O'Rourke, L.; Kiss, Cs.; Barucci, A., et al., Herschel observations of the Hayabusa 2 asteroid $162173\left(1999 \mathrm{JU}_{3}\right)$, European Planetary Science Congress 2012, id. EPSC2012-753 (2012)

24. Ott, S., "The Herschel Data Processing System HIPE and Pipelines Up and Running Since the Start of the Mission", in Astronomical Data Analysis Software and Systems XIX., eds. Y. Mizumoto, K.-I. Morita, \& M. Ohishi, ASP Conf. Ser., 434, 139 (2010)

25. Pál. A., FITSH- a software package for image processing, MNRAS, 421, 1825 (2012)

26. Poglitsch, A, Waelkens, C., Geis, N., et al., The Photodetector Array Camera and Spectrometer (PACS) on the Herschel Space Observatory, A\&A, 518, L2 (2010) Popesso, P., Magnelli, B., Buttiglione, S., et al., The effect of the high-pass filter data reduction technique on the Herschel PACS Photometer PSF and noise, A\&A, submitted, arXiv:1211.4257. (2012)

27. Santos-Sanz, P., Lellouch, E., Fornasier, S., et al., "TNOs are Cool": A survey of the trans-Neptunian region. IV. Size/albedo characterization of 15 scattered disk and detached objects observed with Herschel-PACS, A\&A, 541, A92 (2012)

28. Shirahata, M., Matsuura, S., Hasegawa, S., Calibration and Performance of the AKARI Far-Infrared Surveyor(FIS) - Slow-Scan Observation Mode for PointSources, PASJ, 61, 737 (2009)

29. Stansberry, J., Grundy, W.M., Brown, M.E., et al., in The Solar System Beyond Neptune, Physical Properties of Kuiper Belt and Centaur Objects: Constraints from the Spitzer Space Telescope, p.161 (Tuscon, AZ: Univ. Arizona Press), p. 161 (2008) Vavrek, R., Wetzstein, M. \& Royer, P., Multiresolution deglitching routines for Herschel-PACS data, in Astronomical Data Analysis 5, Heraklion, Crete (2008) 
30. Vilenius, E., Kiss, Cs., Mommert, M., et al., "TNOs are Cool": A survey of the trans-Neptunian region. VI. Herschel/PACS observations and thermal modeling of 19 classical Kuiper belt objects, A\&A, 541, A94 (2012) 